Original article

\title{
Experimental method for burn skin wound modeling in pigs
}

\author{
Sergey B. Bogdanov ${ }^{1,2}$, Anton V. Karakulevi, ${ }^{1,}$ Yuliya A. Bogdanova ${ }^{2}$, Alexander S. Sotnichenko², \\ Irina V. Gilevich ${ }^{1,2}$, Karina I. Melkonyan ${ }^{2}$, Valeriya A. Aladyina ${ }^{1,2}$ \\ ${ }^{1}$ S.V. Ochapovsky Regional Clinical Hospital No.1 \\ ${ }^{2}$ Kuban State Medical University
}

Received - 28 October 2020, Accepted 26 May 2021

Original Text (c) Bogdanov S.B., Karakulev A.V., Bogdanova Yu.A., Sotnichenko A.S., Gilevich I.V., Melkonyan K.I., Aladyina V.A., 2021, published in Saratov Journal of Medical Scientific Research 2021; 17(1): 46-50

(C) 2021, Bogdanov S.B., Karakulev A.V., Bogdanova Yu.A., Sotnichenko A.S., Gilevich I.V., Melkonyan K.I., Aladyina V.A.

(C) 2021, Saratov Medical Journal

\begin{abstract}
:
Objective: to develop an experimental model for studying the course of the wound healing process and the efficacy of applying wound dressings in clean conditions, as well as on a purulent wound on the same animal.

Materials and Methods. Pig wound modeling is used as an in vivo model. In an operating theater, under general anesthesia, a laboratory animal laying on the side was marked with the future burn boundaries. A hot steel contact pad imposed a thermal injury to the skin of a given size and required depth (grades III B and IV sensu the classification by Vishnevsky). Then necrotomy was performed, followed by primary and delayed skin autograft, and use of wound dressings. The subjects of our in vivo study were 4 Landrace pigs, $18-24 \mathrm{~kg}$ each.

Results. With III B burns, scar tissue was formed by day 12 under the decellularized scaffolds. By day 8, epithelialization of the wound was occurring under recellularized scaffolds. With grade IV burns, granulation tissue without aseptic inflammation was formed on day 8 .

Conclusion. The developed method for experimental wound modeling allows analyzing the engraftment of wound dressings on the same animal, both in early and in staged surgical treatment of burns.
\end{abstract}

Keywords: burns, wound dressing, animal model.

Cite as Bogdanov SB, Karakulev AV, Bogdanova YuA, Sotnichenko AS, Gilevich IV, Melkonyan KI, Aladyina VA. Experimental method for burn skin wound modeling in pigs. Saratov Medical Journal 2021; 2(2): e0204.

Correspondence to Sergey B. Bogdanov, Tel.: +7 (918) 650 2857. E-mail: karakulev797@gmail.com

\section{Introduction}

According to the World Health Organization, burn wounds are the 2nd or 3rd most common injuries. In the Russian Federation, according to official statistics, burn injuries are ranked 4th: about 450 thousand people injured per year, of which 100 thousand are treated in a hospital [1]. These data confirm the relevance of improving the medical care provided to this category of patients [2]. Currently, novel biological wound dressings are developed, which could provide much better help to burn victims, along with improved surgical treatment methods [3].

The strict norms of the legislation of our country, regulating the development, safety, and introduction of novel drugs and treatment techniques into clinical practice, have become recently more stringent $[4,5]$. In order to introduce successful laboratory results into the clinical practice, a suitable and effective in vivo experimental model is needed. Experimental modeling on laboratory animals is among current conventional practices [6-10].

Skin autografts of full thickness, providing the best positive cosmetic outcomes, represent the optimal treatment technique [11]. However, granulating wounds limit performing this type of plastic surgery. Experimental work is underway to investigate the engraftment of full-thickness skin autografts [12].

Experimental models undergo constant improved, which benefits more thorough studies of wound healing processes [13-17]. There is a method that allows simulating burn injury under experimental conditions in small experimental animals (mice, rats). The method presumes imposing a burn injury to an animal by immersing the dorsal surface of the body in hot water, the temperature of which ranges from 65 to $100{ }^{\circ} \mathrm{C}$. With this method, wet necrosis is formed. To isolate tissues, subcutaneous injections of $150-200 \mathrm{~mL}$ of air are performed; after thermal injury, the air is expelled.

Another method is a method of modeling a full-thickness skin wound in pigs of Bolshaya Belaya (Big White) breed: six full-thickness skin wounds are protected from marginal epithelialization and retraction by plastic chambers $4 \mathrm{~cm}$ in diameter [18].

The objective of developing a new method was building an experimental model for studying the course of the wound healing process and the efficacy of using wound dressings in clean conditions and on a purulent wound on the same animal. 


\section{Materials and Methods}

This study involved four Landrace pigs (18-24 kg each). An important feature of this breed is low amount of subcutaneous fat. The maintenance of experimental pigs and all manipulations with animals were carried out in compliance with the European Convention for the Protection of Vertebrate Animals Used in Experiments and for Other Scientific Purposes, ETS No. 123 of March 18, 1986; and with the Russian Federation set of technical standards, GOST 34088-2017, Guidelines for Accommodation and Care of Laboratory Animals. Rules for Keeping and Care of Farm Animals, of January 8, 2018, after receiving an approval from the Ethics Committee.

Pig skin, de- and recellularized by fibroblasts obtained from a donor, was used as wound dressings. Decellularization was carried out sensu the original method (Russian Federation Patent Application No. 2019133278/04 (065726), based on the detergent enzymatic protocol via using TrypsinVersene solution (Biolot, Russia), 1\% solution of Triton X-100 (Sigma-Aldrich, USA) and 4\% sodium deoxycholate solution (Sigma-Aldrich, USA) in combination with 0.002M Na2EDTA, porcine pancreatic DNase I (Sigma-Aldrich, USA) The total process duration was 64 hours.

Fibroblasts were isolated by the enzymatic method from the collected native skin samples, which were cultured until passage number 4. Then, static recellularization of decellularized matrices was carried out in a 6-well cell culture plate with a cell suspension at the rate of 25,000 cells $/ \mathrm{cm} 2$ for two days in a $\mathrm{CO} 2$ incubator.

Thermal trauma in animals was performed under operating conditions with endotracheal anesthesia, with animals laying on their side. A ruler was used to mark the boundaries of the future burn. A hot steel contact pad $20 \mathrm{~cm}$ by $5 \mathrm{~cm}$ was employed for imposing the burns. Its contact with skin lasted 1-3 s. The exposure time depended on desired depth (grades III B or IV century sensu the classification by Vishnevsky). On days 1-3 after the thermal injury, all necrotic tissues were removed via necrotomy. As a result, two wound surfaces were formed: a wound, the bottom of which was the reticular layer of the dermis, and the wound surface, the bottom of which was the fascia and muscle fibers (Fig. 1). After the performed necrotomy, stepping back from the edges of the wounds by $1-2 \mathrm{~cm}$ (in order to prevent epithelialization from the edges), wound dressings under study and/or skin grafts were sutured to the bottom, provided that the distance between them was 1-2 cm (Fig. 2). A bandage with Levomekol@ ointment was applied.

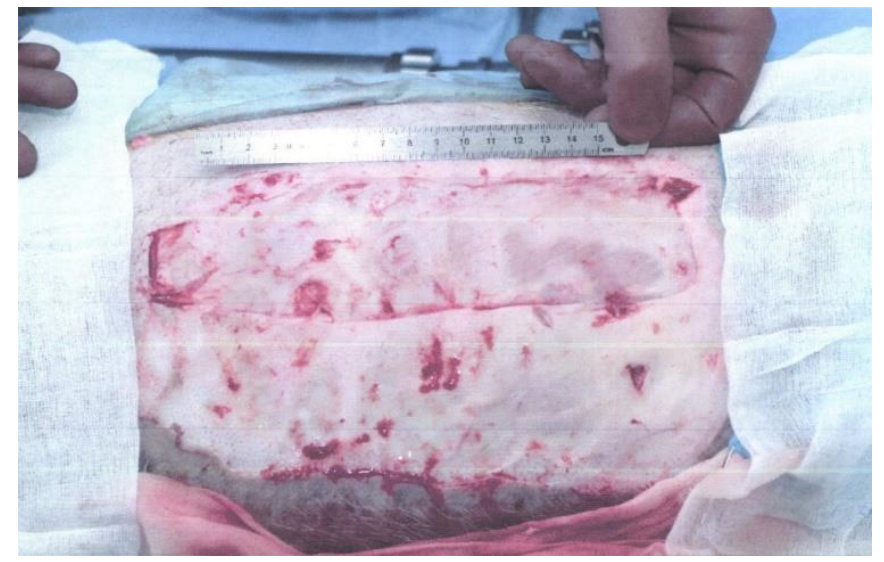

Figure 1. Necrotomy. Formation of wound surfaces of various depths
An emergence of a pig from anesthesia also occurred when it was laying on its side. Each of subsequent 3-4 days after the procedure, a series of biopsies was performed under general anesthesia. After each surgical intervention, ointment dressings were applied. After 7-9 days from the first surgical intervention, a grade IV burn wound sensu Vishnevsky's classification began granulating. After the formation of mature granulation tissue, a tangential excision of granulations to the fibrous layer was performed, followed by suturing of the wound dressings under investigation and/or skin grafts to the bottom of the wound (Fig. 3). This technique allows evaluating the efficacy of investigated dressings on the same pig both on a clen surgical wound, along with a granulating wound. On each of subsequent 3-4 days, a series of biopsies of the investigated dressings and/or skin grafts was performed in order to study the efficacy of their engraftment on the surfaces of burn wounds of various depths in dynamics. Upon completion of the experiment, after 2-3 weeks from imposing the burns, on the remaining wounds that did not epithelialize, free skin grafting was performed with split-thickness autografts $0.3-0.5 \mathrm{~mm}$ thick. The donor material was also taken from the affected side (Fig. 4). After 5-7 days from the last operation, all dressings were removed, and complete closure of the wounds was noted. Experimental animals were removed from the experiment without wound defects (Fig. 5) [19].

For morphological analysis, the samples were fixed in $10 \%$ neutral formalin, dehydrated, and then embedded in paraffin using Leica TP1020 tissue processor (Germany), in accordance with the standardized technique. Sections $5 \mu \mathrm{m}$ thick were stained with hematoxylin and eosin (SigmaAldrich, USA) according to the conventional technique. The study of micropreparations was carried out on Olympus CX 41 microscope (Japan).

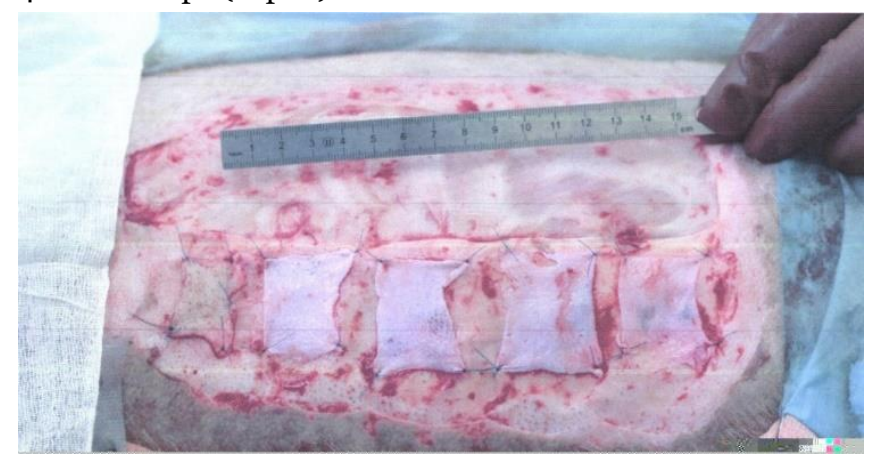

Figure 2. Applying and fixing the test materials to the lower layers of the dermis

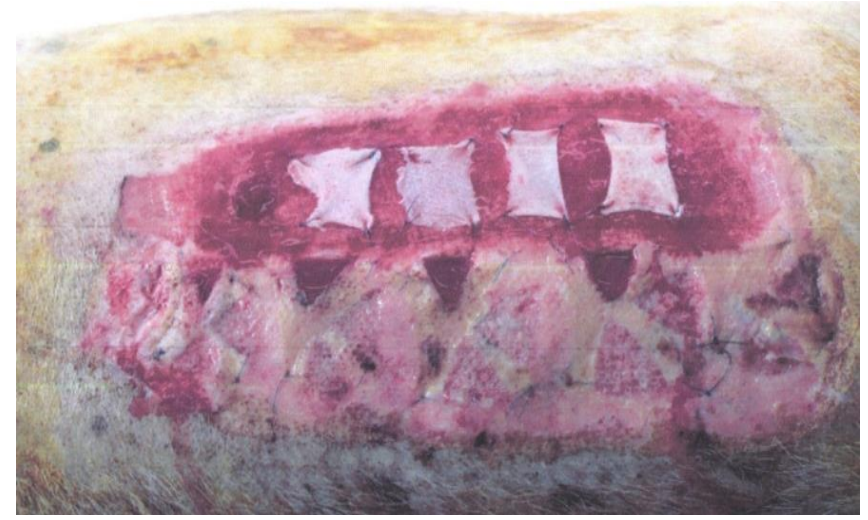

Figure 3. Applying and fixing the test materials to the fibrous layer of the granulation tissue 


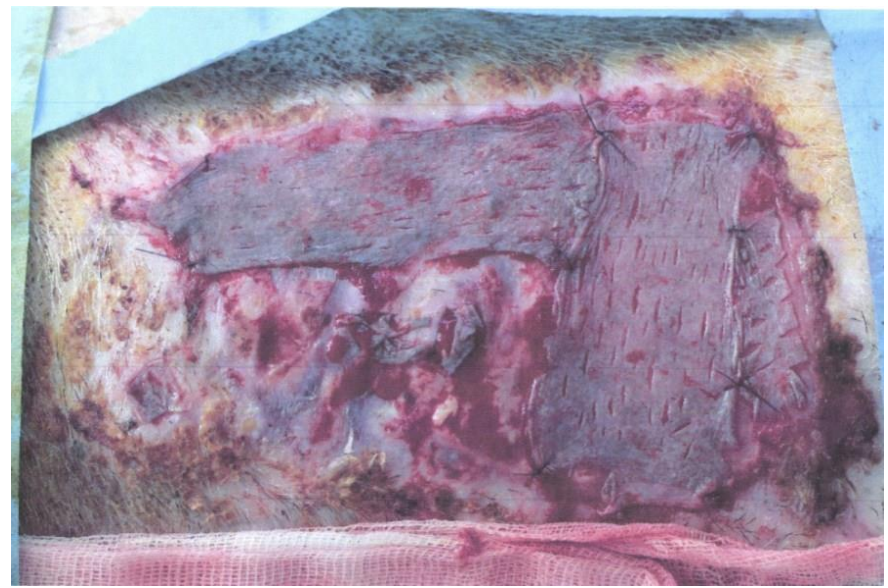

Figure 4. Skin autograft wound closure

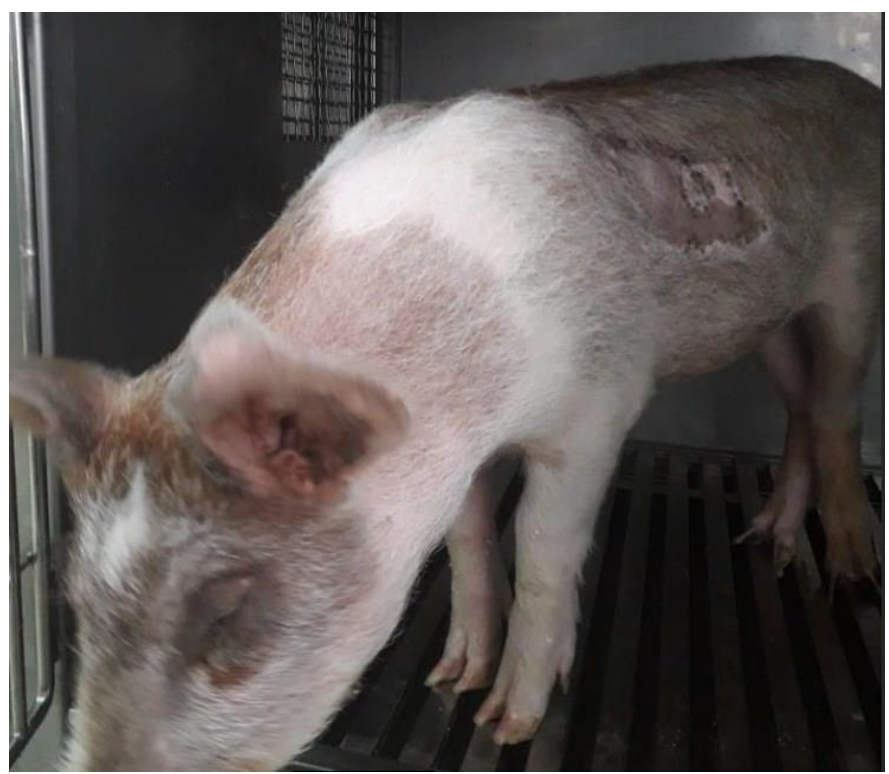

Figure 5. Animal without wound defects after three months

\section{Results}

Observing experimental animals in the vivarium, and performing all manipulations under general anesthesia (imposing burns, performing surgical interventions, and taking biopsy materials) made it possible to save the life of each animal and, upon withdrawal from the experiment, to perform skin autograft to ensure the complete absence of wounds.

The study noted morphological differences among the bottoms of the wounds, closed by de- or recellularized matrices in the III B grade wound sensu Vishnevsky.

After implanting acellular dermis, a mild aseptic inflammatory reaction with lymphoid and macrophage infiltration and saturation of the matrix with fibrin and erythrocytes was observed in the underlying tissues. On day 12, the wound dressing, represented by decellularized material, was becoming dry and dense. As a result, the wound dressing was detaching from the wound surface, and scar tissue was forming its place. The scar tissue required excision and skin autografting with a split-thickness skin graft on day 20.
In case of using dermal matrix, recellularized by the fibroblasts from a donor, as a wound dressing, the morphological and clinical results were significantly different. The recellularized matrix adhered well to the bottom of the wound and was taken into the fold by palpation. Morphologically, on days 2-5, a mild aseptic inflammatory reaction was also developing, but it occurred solely in the upper sections of the wound, adjacent to the matrix.

On day 8, a pronounced proliferation of dermal fibroblasts was starting under the recellularized matrix. By that time, the formation of flat non-keratinizing epidermis from proliferating appendages of the skin was occurring. When using such matrix, there were no indications for performing skin autografts.

The course of the wound healing process in a grade IV wound sensu Vishnevsky's classification in the case of using both of described types of wound dressings occurred without substantial differences. In the course of wound healing under the scaffolds, the growth of granulation tissue and the development of aseptic inflammation were observed. Strong adhesion of both decellularized and recellularized scaffolds to the surface did not take place; on days 5-8, wound dressings were detached without effort, exposing the granulating wound surface.

\section{Discussion}

The described method of experimental burn wound modeling in pigs allows determining the efficacy of using dressings on burn wound surfaces of various depths with great accuracy. Forming a wound in the study of various wound dressings with the condition of stepping $2 \mathrm{~cm}$ away from the wound edges helps preventing marginal epithelialization, and, on wounds of the same depth, to reliably study the course of the wound healing process with various types of autografts and various dressings.

We propose good prospects of using de- and recellularized dermal matrices in the case of their application to shallow wounds. Use of these dressings on granulating wounds made it possible to avoid infection, but did not serve a basis for the complete skin restoration. We attribute this to the lack of skin appendages necessary for the regeneration of epidermis.

Performing the proposed technique is possible in the presence of a vivarium, veterinarian, anesthesiologist and combustiologist. Observation of the animal is carried out dynamically just for a month, but this makes it possible to fully assess the course of the wound healing process both in early surgical treatment of burns and in staged surgical treatment, with an opportunity to study different ways of treating burn wounds of various depths on the same animal.

Comparing the described method with its closest analogs, we should point out a number of disadvantages of the method for experimental modeling of thermal injury wound surfaces in laboratory mice or rats by immersing their dorsal body surface in water, the temperature of which ranges $65^{-100}{ }^{\circ} \mathrm{C}$ : (1) difficulty of applying the method to large laboratory animals, such as pigs; (2) impossibility of avoiding thermal damage of deep tissues when simulating a skin burn; (3) formation of wet skin necrosis; (4) difficulty of isolating deep tissues from the exposure to high temperatures via a subcutaneous injection of $150-200$ milliliters of air at the time of burn injury, followed by its expelling; also, this manipulation does not allow to fully transfer this model to 
the situation of receiving thermal injury in humans; (5) difficulty of forming a wound of a given size [20].

The disadvantages of the method for modeling a fullthickness skin wound in a female pig of the Bolshaya Belaya breed, with six full-thickness wounds protected from marginal epithelialization and retraction by plastic chambers $4 \mathrm{~cm}$ in diameter, include: (1) imperfection of protection from marginal epithelialization by plastic chambers; (2) involuntary traumatization of formed wounds by animals; (3) impossibility of creating thermal injury wounds of different depths; (4) impossibility of an objective assessment of efficacy of studied biomaterials for burn wounds of various depths; 5) placement of wound surfaces in more than one area of the animal's body surface [18].

\section{Conclusion}

The method we have developed for experimental wound modeling allows providing an objective efficacy assessment of original wound dressings and conducting a comparative characterization of the wound healing process course. Furthermore, the method is devoid of disadvantages that the closest analogs have.

\section{Conflict of Interest: none declared.}

Funding. The article was prepared within the framework of the complex research project, "Cellular Mechanisms of Tissue Regeneration. Development of Tissue-Engineered Structures Using Biological and Synthetic Scaffolds" (Registration number AAAA-A16-116042550089-5 of 25 April, 2016).

\section{References}

1. Ostrovsky NV, Kuspits EV. Problems of organizing first aid and interaction with a specialized burn center. In: Novel Technologies of Medical Care. Proceedings of the scientific and practical conference. Moscow, 2016; 22-3. [In Russian].

2. Bogdanov SB, Kurinny NA, Polyakov AV, et al. The Method of Skin Graft After Early Necrotomy. The patent for the invention RU 2295924 C1, 27 March, 2007. Application No. 2005123211/14 of 21 July, 2005. [In Russian].

3. Bogdanov SB, Afaunova ON. Using wound closures in surgical treatment of partial thickness burns on the limbs in functionally active areas. Postgraduate Doctor 2016; 79 (6): 4-9. [In Russian].

4. Climov M, Medeiros E, Farkash EA, et al. Bioengineered selfassembled skin as an alternative to skin grafts. Plastic and Reconstructive Surgery Global Open 2016; 4 (6): e731. https://doi.org/10.1097/GOX.0000000000000723

5. MacNeil S. Progress and opportunities for tissue-engineered

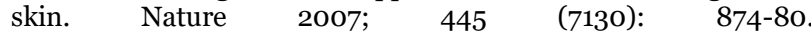
https://doi.org/10.1038/natureo5664

6. Burd A, Ahmed K, Lam S, et al. Stem cell strategies in burns care Burns 2007; 33 (3): 282-291. https://doi.org/10.1016/j.burns.2006.08.031

7. Chua A. WC, Khoo YC, Tan BK, et al. Skin tissue engineering advances in severe burns: Review and therapeutic applications. Burns \&amp; trauma 2016; 4 (1): 3. https://doi.org/10.1186/s41038-016-0027-y

8. Leclerc T, Thepenier C, Jault P, et al. Cell therapy of burns. Cell proliferation 2011; (44): 48-54. https://doi.org/10.1111/j.13652184.2010.00727.x

9. Golinski P, Menke H, Hofmann M, et al. Development and characterization of an engraftable tissue-cultured skin autograft: Alternative treatment for severe electrical injuries. Cells Tissues

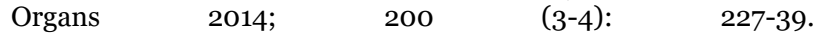
https://doi.org/10.1159/000433519
10. Zeller N, Valesky E, Butting M, et al. Clinical application of a tissue-cultured skin autograft: An alternative for the treatment of non-healing or slowly healing wounds? Dermatology 2014; 229 (3): 190-8. https://doi.org/10.1159/000362927

11. Bogdanov SB, Babichev RG. Method of Skin Autograft Sampling. Patent for invention RU 2618166 C, 02.05.2017. Application No. 2016103838 of o5 February, 2016. [In Russian].

12. Bogdanov SB, Babichev RG, Marchenko DN, et al. Plastic surgery with full-layer skin autografts for the wounds of various etiologies. Innovative Medicine of the Kuban Region 2016; (1): 30-7. [In Russian].

13. Climov M, Medeiros E, Farkash EA, et al. Bioengineered selfassembled skin as an alternative to skin grafts. Plastic and Reconstructive Surgery Global Open 2016; 4 (6): e731. https://doi.org/10.1097/GOX.0000000000000723

14. Keck M, Haluza D, Lumenta DB, et al. Construction of a multilayer skin substitute: Simultaneous cultivation of keratinocytes and preadipocytes on a dermal template. Burns 2011; 37 (4): 626-30. https://doi.org/10.1016/j.burns.2010.07.016

15. Wormald JC, Fishman JM, Juniat S. Regenerative medicine in otorhinolaryngology. J Laryngol Otol 2015; 129 (8): 732-9. https://doi.org/10.1017/So022215115001577

16. Gallico GG the 3rd, O'Connor NE, Compton CC, et al. Permanent coverage of large burn wounds with autologous cultured human epithelium. New England Journal of Medicine 1984; 311 (7): 44851. https://doi.org/10.1056/NEJM198408163110706

17. Van der Veen VC, van der Wal MB, van Leeuwen MC, et al. Biological background of dermal substitutes. Burns 2010; 36 (3): 305-21. https://doi.org/10.1016/j.burns.2009.07.012

18. Shevchenko RV, James SE, Reed MJ, et al. Experimental pig model as an effective tool for transferring scientific knowledge to the clinic to replenish the combustiologist's arsenal. Combustiology 2007; (30) [In Russian].

19. Bogdanov SB, Karakulev AV, Porkhanov VA, et al. The Method of Experimental Skin Wound Modeling in Pigs. Patent for invention RU 2726600 C1, 14.07.2020. Application No. 2019122965 of 16 July, 2020.

20. Andreev SV. Modeling of Diseases. Moscow: Medicine, 1973; 336 p. [In Russian].

\section{Authors:}

Sergey B. Bogdanov - Head of Burn Unit, Research InstituteProfessor S.V. Ochapovsky Regional Clinical Hospital No.1; DSc, Associate Professor, Department of Surgery No.1, School of Advanced Training and Professional Retraining, Kuban State Medical University, Krasnodar, Russia;

Anton V. Karakulev - Traumatologist and Orthopedist, Burn Unit, Research Institute - Professor S.V. Ochapovsky Regional Clinical Hospital No.1; Graduate Student, Department of Surgery No.1, School of Advanced Training and Professional Retraining, Kuban State Medical University, Krasnodar, Russia;

Yuliya A. Bogdanova - PhD, Assistant Professor, Department of General and Clinical Pathophysiology, Kuban State Medical University, Krasnodar, Russia;

Alexander S. Sotnichenko - PhD, Head of Laboratory Unit on Basic Research in Regenerative Medicine, Central Research Laboratory, Kuban State Medical University, Krasnodar, Russia;

Irina V. Gilevich - PhD, Head of Laboratory on Developing and Studying Novel Technologies of Disease Treatment, Research Institute - Professor S.V. Ochapovsky Regional Clinical Hospital No.1; Instructor, Department of Oncology with Course on Thoracic Surgery, School of Advanced Training and Professional Retraining, Kuban State Medical University, Krasnodar, Russia;

Karina I. Melkonyan - PhD, Head of Laboratory on Basic Research, Central Research Laboratory, Kuban State Medical University, Krasnodar, Russia;

Valeriya A. Aladyina - Polyclinic Surgeon, Research Institute Professor S.V. Ochapovsky Regional Clinical Hospital No.1; Graduate Student, Department of Surgery No.1 School of Advanced Training and Professional Retraining, Kuban State Medical University, Krasnodar, Russia. 